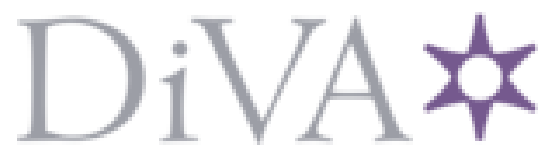

http://www.diva-portal.org

This is the published version of a paper published in Scandinavian Journal of Psychology.

Citation for the original published paper (version of record):

Lundqvist, L-O. (1995)

Facial EMG reactions to facial expressions: a case of facial emotional contagion?.

Scandinavian Journal of Psychology, 36(2): 130-141

https://doi.org/10.1111/j.1467-9450.1995.tb00974.x

Access to the published version may require subscription.

N.B. When citing this work, cite the original published paper.

Permanent link to this version:

http://urn.kb.se/resolve?urn=urn:nbn:se:oru:diva-55058 


\title{
Facial EMG reactions to facial expressions: A case of facial emotional contagion?
}

\author{
LARS-OLOV LUNDQVIST \\ Department of Psychology, Uppsala University, Uppsala, Sweden
}

Lars-Olov Lundqvist (1995). Facial EMG reactions to facial expressions: A case of facial emotional contagion? Scandinavian Journal of Psychology, 36, 130-141.

The purpose of this study is to explore whether subjects exposed to stimuli of facial expressions respond with facial electromyographic (EMG) reactions consistent with the hypothesis that facial expressions are contagious. This study further examines whether males and females differ in facial EMG intensity. Two experiments demonstrated that subjects responded with facial EMG activity over the corrugator supercilii, the zygomatic major, the lateral frontalis, the depressor supercilii, and the levator labii muscle regions to stimuli of sad, angry, fearful, surprised, disgusted and happy faces, that, to large extent, were consistent with the hypothesis that facial expressions are contagious. Aspects of gender differences reported in earlier studies were found, indicating a tendency for females to respond with more pronounced facial EMG intensity.

Key words: Facial reaction, electromyography, facial expression, emotional contagion, mimicry, gender difference.

Lars-Olov Lundquist, Department of Psychology, Uppsala University, Box 1854, S-751 48 Uppsala, Sweden

\section{GENERAL INTRODUCTION}

Charles Darwin (1872) argued that facial expressions of emotion have an adaptive value in social communication because they reveal something about the inner state of the responder that is observable to others, and that this communicative aspect of facial expressions was a major force in shaping their evolution. A phenomenon observed by Darwin (1872), as well as by several others during the last two centuries, is the tendency by which people in social situations imitate or mimic the facial expressions of others' (e.g., McDougall, 1928; Marañon, 1950; Ribot, 1897; Smith, 1759/1976; Spencer, 1881). These observations suggest that people can "catch" the emotions expressed by others, a phenomenon that recently has been conceptualized as emotional contagion (Hatfield et al., 1992).

Empirical results consistent with the hypothesis that facial expressions are contagious have been found in studies investigating facial electromyographic (EMG) reactions of subjects exposed to pictures of emotional facial expressions (Dimberg, 1982, 1988, 1990a, 1991; Dimberg \& Christmanson, 1991; Dimberg \& Lundqvist, 1990). In these studies, it was revealed that, pictures of angry faces evoked increased EMG activity from the corrugator supercilii muscle region compared to pictures of happy faces, that in their turn evoked increased EMG activity from the zygomatic major muscle region. The corrugator supercilii muscle is used when frowning, whereas the zygomatic major muscle is contracted when smiling (Hjortsjö, 1970).

These studies were concerned with only two facial muscles and two facial expressions of emotion. To conclude that facial emotional contagion is a general phenomenon in face-toface situations, it is important to demonstrate that subjects mimic other stimuli in addition to happy and angry facial expressions, such as faces expressing fear and surprise. 
Moreover, there are results showing that females tend to be more facially expressive and to have more pronounced facial reactions than males (Dimberg, 1990b; Dimberg \& Lundqvist, 1990; Schwartz et al., 1980). Females also tend to score higher in nonverbal receiving ability compared to males (for reviews, see Buck, 1984; Hall 1978). These results suggest that females are more susceptible to facial emotional contagion.

To investigate these questions, two experiments were conducted. In both experiments male and female subjects were presented to pictures of facial expressions while the EMG activity from the facial muscle regions that are most important when forming these facial expressions were recorded.

\section{EXPERIMENT 1}

In the present experiment subjects were exposed to pictures of faces expressing fear, anger, surprise, and happiness. Based on the hypothesis that facial expressions are contagious, it was predicted that stimuli of faces expressing fear and anger should evoke more intense EMG activity from the muscle region of corrugator supercilii (knits the brow), and that the stimuli of angry faces in addition should elicit more intense activity from the depressor supercilii (pulls the brow in and down) muscle region. Furthermore, stimuli of faces expressing surprise were expected to evoke more intense EMG activity from the muscle region of lateral frontalis (raises the outer brow). Finally, stimuli of happy faces were predicted to elicit more intense EMG activity from the zygomatic major (pulls the lip corner up and back) muscle region (Hjortsjö, 1970).

In the study by Dimberg and Lundqvist (1990), where only stimuli of happy and angry facial expressions were used, females showed more pronounced facial muscle reactions compared to males. That is, females responded with greater zygomatic major activity to happy faces compared to males. In addition, females differed between angry and happy faces in both corrugator supercilii and zygomatic major, whereas males only differed between angry and happy faces in corrugator supercilii. Facial expressions displayed by female stimulus persons did not evoke more intense facial muscle reactions than facial expressions displayed by male stimulus persons. However, with the extended number of different facial stimuli and facial muscle recording sites in the present experiment, an interaction between sex of the subject and sex of the stimulus persons can not be ruled out. Therefore, as in the study by Dimberg and Lundqvist (1990), sex was manipulated in a $2 \times 2$ factorial design by exposing male and female subjects to facial expressions of both male and female stimulus persons.

\section{Method}

Subjects. Forty-eight psychology students (24 males and 24 females) at Uppsala University participated in the experiment.

Apparatus and data scoring. The subjects were tested individually in a sound attenuated chamber by exposing them to slides selected from Ekman and Friesen's (1976) "Pictures of facial affect". The slides were pictures of males and females displaying angry, happy, fearful, and surprised facial expressions. The slides were projected onto a screen $1.5 \mathrm{~m}$ in front of the subject, producing a picture of $30 \times 45 \mathrm{~cm}$. The exposure time was set at $8 \mathrm{~s}$ and controlled by an electronic timer. All equipment was placed outside the chamber.

Bipolar EMG recording from the left side of the subject's face were made with miniature surface $\mathrm{Ag} / \mathrm{AgCl}$ electrodes filled with Beckman electrode paste. Before attaching the electrodes, the skin was cleaned with alcohol dipped cotton-tops and then rubbed with electrode paste to decrease inter-electrode impedance; all interelectrode impedance was reduced to less than $10 \mathrm{k} \Omega$. The electrodes were placed over the corrugator supercilii, the zygomatic major, the depressor supercilii, and the lateral frontalis muscle regions according to Fridlund and Cacioppo's (1986) "Guidelines for Human Electromyographic Research". However, because of limited space, the recordings of the depressor supercilii and the corrugator supercilii muscle regions had to share one electrode (Fridlund \& Cacioppo, 1986). 
The electrodes were connected to Coulbourn Hi Gain Bioamplifiers with low-pass filters set at $1000 \mathrm{~Hz}$ and high-pass filters set at $10 \mathrm{~Hz}$. Notch filters at $50 \mathrm{~Hz}$ were installed to diminish interference from the electric mains. The raw EMG signals were analyzed by Coulbourn contour following integrators with the time constant set at $0.02 \mathrm{~s}$. The output signal was digitized on-line at $100 \mathrm{~Hz}$ per channel by a Coulbourn 12 Bit A/D converter and stored on an IBM XT. All analyses were performed on arbitrary data units. The data were scored as phasic responses; i.e., the mean EMG during $1 \mathrm{~s}$ immediately before stimulus onset was subtracted from that recorded during the stimulus exposure. Thus, both positive and negative responses are evaluated.

Procedure. The subjects were told that the purpose of the experiment was to measure physiological reactions to facial stimuli. To reduce the subjects' attention to their facial muscles, they were told a cover story that the electrodes were measuring sweat gland activity (Dimberg, 1982).

Each subject was exposed to eight different stimulus persons. That is, each subject was exposed to one angry, one happy, one fearful, and one surprised face expressed by four different males as well as one angry, one happy, one fearful, and one surprised face expressed by four different females. Different subjects saw different stimulus persons. The order of presentation of male and female stimulus persons as well as the order of presentation due to the emotional expressions of the stimuli were counterbalanced among subjects. Each slide was exposed for $8 \mathrm{~s}$ in 6 adjacent trials with an intertrial interval varying between 25 to $45 \mathrm{~s}$.

Before leaving the laboratory the subjects were interviewed to confirm that the cover story had succeeded. Two subjects were aware that their facial muscle activity was measured and they were excluded from the analysis.

Design and statistical analysis. Before analysis, data were collapsed over trials within each stimulus type. Hence, the design was a $2 \times 2 \times 4$ split-plot (Kirk, 1968) with Sex of subject (males vs. females) as between-subjects factor and Sex of stimulus (males vs. females) and Stimulus (fearful, angry, surprised. and happy facial expressions) as within-subject factors. The statistical analysis was performed by a MANOVA with Pillai-Bartlett $V$ as test statistics (Olson, 1976), followed by ANOVAs, with GeisserGreenhouse correction of degrees of freedom, on each muscle separately. The comparisons among means were evaluated by Tukey HSD, with $\alpha=0.05$.

\section{Results and discussion}

The omnibus MANOVA on facial EMG data revealed a significant effect of Stimulus, $V=0.3569, F(12,411)=4.62, p<0.0001$, which indicates that the facial expressions of stimuli evoked different facial muscle response patterns. No significant main or interaction effects of Sex of subject and Sex of stimulus were observed. Given these results, the Stimulus effects of the univariate ANOVAs on each muscle were examined.

The ANOVA for corrugator supercilii revealed a significant effect of Stimulus, $F(2.7,126.1)=7.63, p=0.0002$, MSe $=0.0154$. As can be seen in Fig. 1, all stimuli evoked

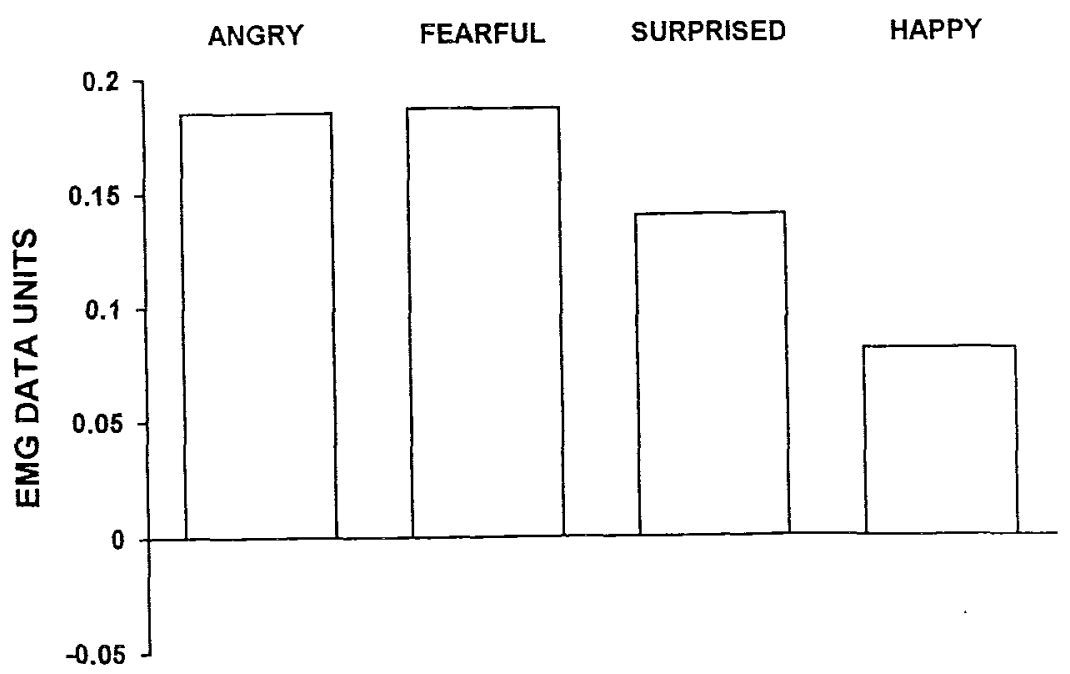

Fig. 1. The mean facial EMG response from the corrugator supercilii muscle region to stimuli of facial expressions obtained in Experiment 1. 
increased EMG activity. However, as expected from the facial emotional contagion hypothesis, stimuli of faces expressing fear and anger evoked significantly larger EMG responses compared to happy faces, as indicated by the HSD test (HSD $=0.066$, df 4,126 ). The EMG responses to stimuli of faces expressing surprise did not differ significantly from the other stimuli.

The ANOVA for zygomatic major showed a significant effect of Stimulus, $F(2.0,93.0)=$ $8.69, p=0.0004, \mathrm{MSe}=0.0330$. The means are illustrated in Fig. 2. Comparisons among means revealed that happy faces, as predicted, evoked significantly larger responses compared to faces expressing anger and fear, as indicated by the HSD test (HSD $=0.097$, df 4, 93). However, not inherent in the hypothesis that facial expressions are contagious, stimuli of faces expressing surprise evoked significantly larger responses compared to angry faces. This result may be explained by a difference in stimulus valence. That is, stimuli of faces expressing surprise may be perceived as more pleasant than stimuli of angry facial expressions (Lundqvist \& Dimberg, unpublished data).

Furthermore, the ANOVA for depressor supercilii revealed a significant effect of Stimulus, $F(2.5,116.0)=5.17, p<0.0040, \mathrm{MSe}=0.0079$. The means are illustrated in Fig. 3. As

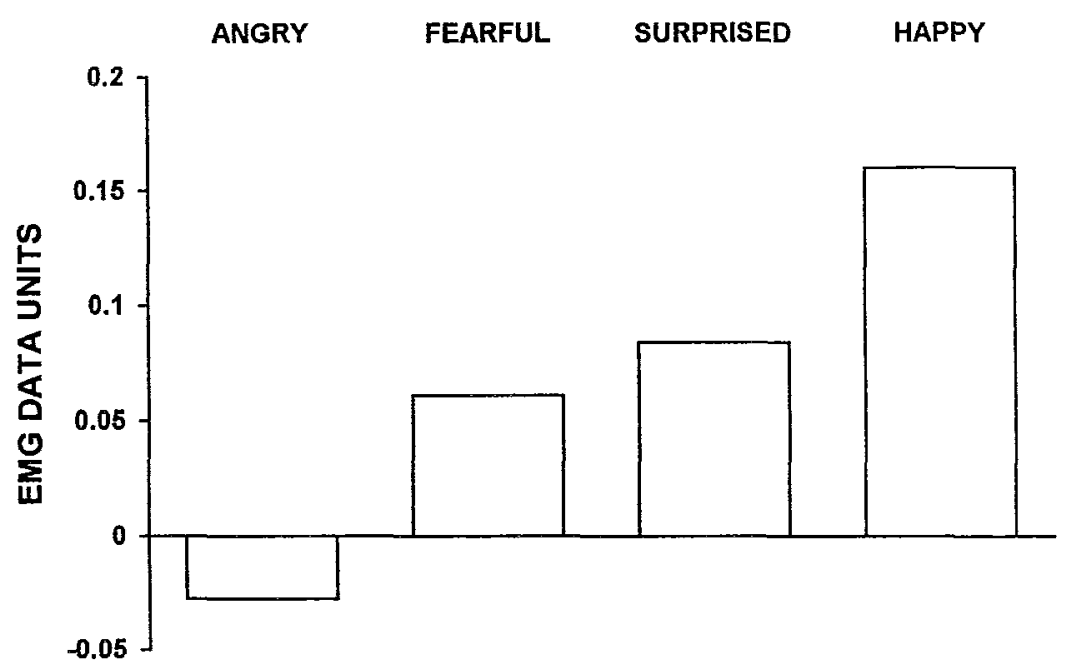

Fig. 2. The mean facial EMG response from the zygomatic major muscle region to stimuli of facial expressions obtained in Experiment 1.

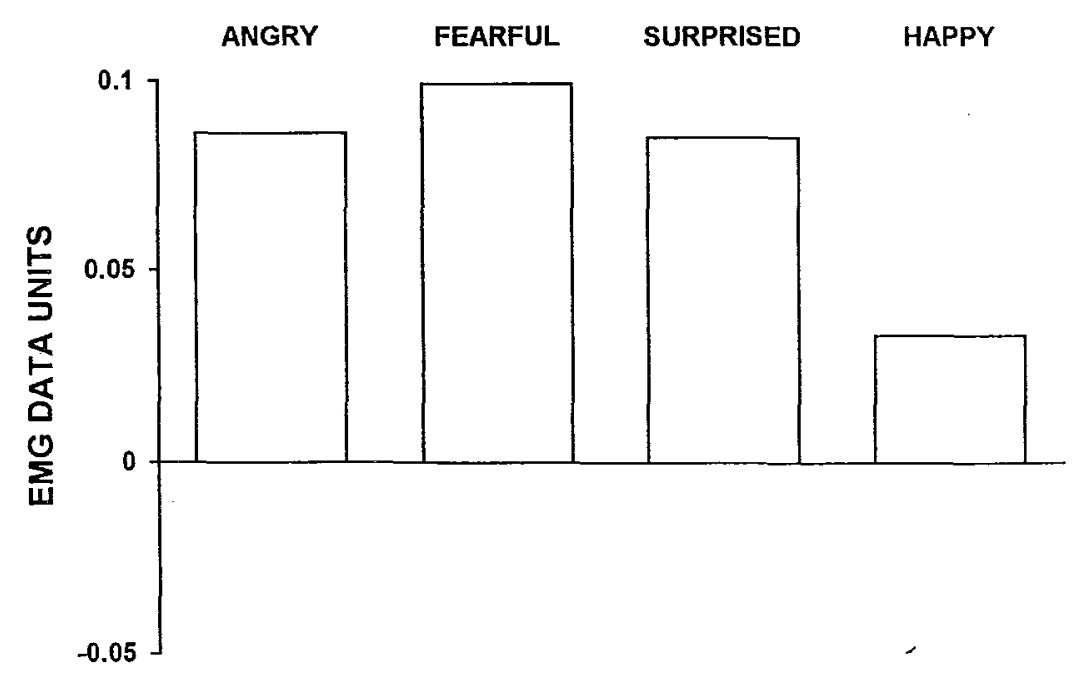

Fig. 3. The mean facial EMG response from the depressor supercilii muscle region to stimuli of facial expressions obtained in Experiment 1. 
indicated by the HSD test ( $\mathrm{HSD}=0.047$, df 4,116 ), stimuli of faces expressing anger, fear, and surprise did not differ significantly among one another, but these stimuli evoked significantly larger responses compared to stimuli of happy facial expressions. Only angry faces were predicted to show increased activity from the depressor supercilii muscle region. However, it should be noticed that the recordings of the depressor supercilii and the corrugator supercilii muscle regions shared one electrode. Such a procedure, and that these two muscle regions are near to each other, may increase the risk of inter-site crosstalk. In fact, the reactions from the depressor supercilii muscle were highly correlated with the corrugator supercilii muscle reactions $\left(r_{x y}=0.85, p<0.001\right)$. This correlation may indicate crosstalk with the corrugator supercilii muscle, suggesting that the recorded EMG activity from the depressor supercilii muscle region should be interpreted cautiously (see Fridlund \& Cacioppo, 1986).

Finally, the ANOVA for lateral frontalis showed a significant effect of Stimulus, $F(2.5,116.4)=5.13, p=0.0042, \mathrm{MSe}=0.0128$. As shown in Fig. 4 , faces expressing surprise and fear evoked increased EMG activity. As expected from the facial emotional contagion hypothesis, faces expressing surprise evoked significantly larger responses compared to happy and angry faces, as indicated by the HSD test (HSD $=0.060$, df 4,116 ). Unexpectedly, faces expressing fear evoked significantly larger responses compared to happy faces. This result may indicate that the electrodes attached over the lateral frontalis muscle region may have picked up some activity from the medial part of the frontalis muscle, which normally is contracted when forming a fear facial expression (Hjortsjö, 1970). On the other hand, as indicated by the original ratings (Ekman \& Friesen, 1976), stimuli of fear facial expressions were perceived to include a component of surprise, though small, which may have produced the obtained EMG activity from the lateral frontalis muscle region.

The omnibus MANOVA showed no significant main or interaction effects including Sex of subject or Sex of stimulus, thus indicating no overall gender differences. However, to more specifically compare the present results with those obtained in the study by Dimberg and Lundqvist (1990), a separate analysis was performed on the present data for corrugator supercilii and zygomatic major muscle activity to stimuli of happy and angry facial expressions. The results supported earlier results on some aspects of gender differences in facial EMG response patterns as indicated by a marginally significant Muscle by Stimulus by Sex of subject interaction $(F(1,46)=3.51, p=0.0675$, MSe $=0.1004)$. Females responded with

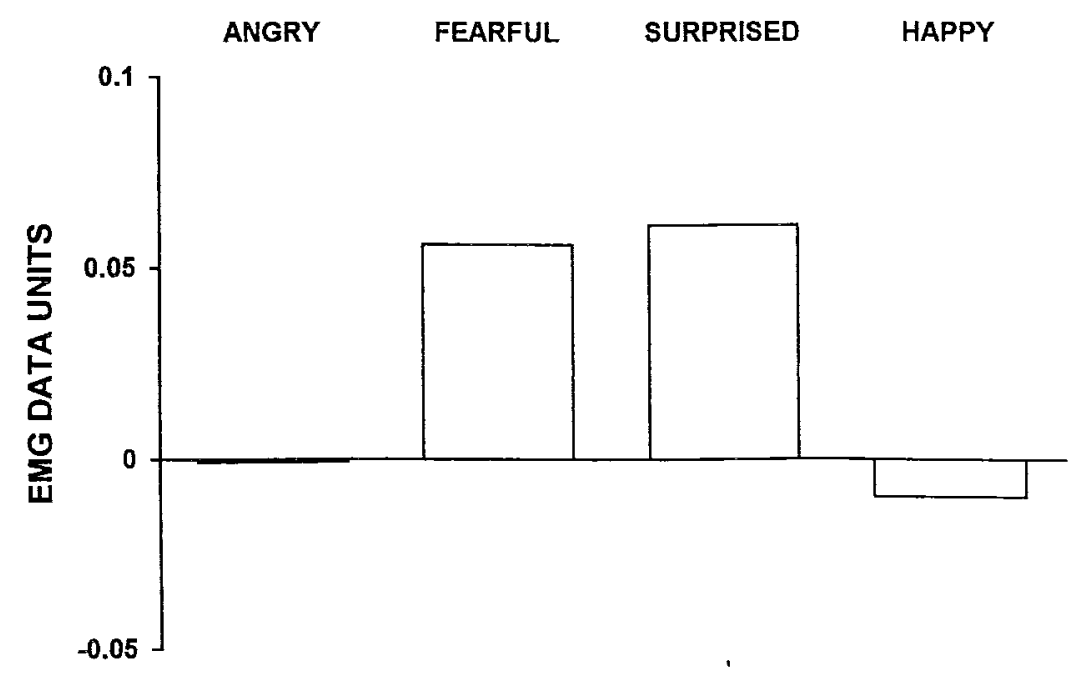

Fig. 4. The mean facial EMG response from the lateral frontalis muscle region to stimuli of facial expressions obtained in Experiment 1. 
more intense corrugator supercilii muscle activity to angry versus happy faces $(t(46)=2.58$, $p<0.05)$ and more intense zygomatic major muscle activity to stimuli of happy compared to angry faces, $(t(46)=4.61, p<0.05)$, whereas males did not $(t(46)=1.03, p>0.05$, and $t(46)=1.93, p>0.05$, respectively).

To summarize Experiment 1, it was demonstrated that stimuli of faces expressing anger, fear, surprise and happiness evoked facial EMG reaction patterns consistent with the hypothesis that facial expressions are contagious. However, only some aspects of gender difference in facial EMG intensity consistent with earlier research was obtained. Not inherent in the facial emotional contagion hypothesis, faces expressing surprise evoked greater zygomatic major activity than angry faces. However, it is important to note that, consistent with the facial emotional contagion hypothesis, stimuli of faces expressing surprise concurrently evoked greater lateral frontalis activity than angry faces as well. Finally, probably due to crosstalk between the corrugator supercilii and the depressor supercilii muscle regions, it was not possible to conclude whether stimuli of angry faces evoke different facial EMG reaction patterns than stimuli of faces expressing fear.

\section{EXPERIMENT 2}

According to research on cross-cultural similarity of facial expression there are at least six universal facial expressions of emotions, i.e., sad, angry, fearful, surprised, disgusted, and happy (Ekman, 1972, 1989; Fridlund et al., 1987). These findings corroborate Darwin's (1872) assumption that facial expressions have a biological basis, suggesting that these universally expressed and recognized facial expressions of emotion are important in social emotional communication, and thus of particular interest from a facial emotional contagion perspective. Hence, the major aim of the present experiment was to investigate the facial EMG reaction patterns from subjects exposed to stimuli of all six facial expressions. Experiment 2 was similar to Experiment 1. However, due to the addition of the stimuli of facial expressions of disgust and the indications of crosstalk with corrugator supercilii, the EMG activity from the muscle region of levator labii (raises the upper lip and widen the nostrils; Fridlund \& Cacioppo, 1986) was recorded instead of the depressor supercilii muscle region.

Based on the hypothesis that facial expressions are contagious, it was predicted that stimuli of sad, angry, and fearful faces should evoke increased EMG activity from the corrugator supercilii muscle region, whereas stimuli of happy facial expressions should evoke increased EMG activity from the zygomatic major muscle region. Furthermore, it was predicted that stimuli of faces expressing disgust should evoke increased activity from the levator labii muscle region and, finally, that stimuli of faces expressing surprise should evoke increased EMG activity from the lateral frontalis muscle region (Hjortsjö, 1970).

However, it can be proposed that subjects may show some muscle reactions due to the fact that a picture is being exposed rather than to the facial expression of stimuli. A stimulus of neutral facial expressions is neutral in respect to facial mimicry but not to exposure artifacts. To reduce possible exposure artifacts, the muscle reactions to neutral faces can serve as a base line to the muscle reactions to stimuli of emotional facial expressions. Therefore, the subjects were also exposed to stimuli of neutral faces and the statistical analyses were performed on difference scores with the phasic mean facial EMG response to stimuli of neutral facial expressions subtracted from the phasic mean facial EMG response to stimuli of emotional facial expressions.

With the introduction of additional stimuli of facial expression and the muscle region of levator labii, Experiment 2 was designed to further investigate whether males and females differ in facial EMG intensity. 


\section{Method}

Subjects. Fifty-six psychology students ( 28 males and 28 females) at Uppsala University participated in the experiment.

Apparatus. The arrangement was identical with that in Experiment 1, except for the stimulus material. In the present experiment subjects were exposed to slides of males and females producing sad, angry, fearful, surprised, disgusted, happy, and neutral facial expressions selected from Ekman and Friesen's (1976) "Pictures of facial affect". The facial EMG electrodes were attached over the corrugator supercilii, the zygomatic major, and the lateral frontalis, and the levator labii muscle regions (Fridlund \& Cacioppo, 1986).

Procedure. The same instruction and cover story as in Experiment $l$ were used. None of the subjects reported that they were aware that their facial muscle activity was measured.

The subjects were exposed to seven different slides of facial expressions (i.e., sad, angry, fearful, surprised, disgusted, happy, and neutral faces) in a balanced order. For a given subject, one stimulus person appeared only in one type of facial expression. Different subjects saw different stimulus persons. Because of the limited number of male persons in the stimulus materials (six male and eight female persons), it was not possible to expose each subject to seven different facial expressions without having to present the same male stimulus person at least twice. Therefore, half of the subjects were exposed to three different male and four different female stimulus persons, and vice versa for the other half of the subjects. This procedure allows for the use of all stimulus persons available in the stimulus material, which will diminish the risk that the EMG data rely on a few specific stimulus persons. However, this procedure makes the sex of the stimulus persons confounded with the facial expression of the stimulus. Therefore, and since neither in the study by Dimberg and Lundqvist (1990) nor in Experiment 1 the sex of the stimulus persons had any effect on facial EMG activity, the sex of stimulus factor was not investigated in the present experiment.

As in Experiment 1, each slide was exposed for $8 \mathrm{~s}$ in 6 adjacent trials with an inter-trial interval varying between 25 to $45 \mathrm{~s}$. Before any analysis was carried out, the data were scored as phasic responses; i.e., the mean EMG during $1 \mathrm{~s}$ immediately before stimulus onset was subtracted from that recorded during the stimulus exposure. Thereafter, the data were collapsed over trials within each stimulus type. Finally, difference-scores between each stimulus of emotional facial expression and the stimulus of neutral facial expression were calculated.

Design and statistical analysis. The design was a $2 \times 6$ split-plot (Kirk, 1968), with Sex of subject (male vs. female) serving as the between-subjects factor and Stimulus (sad, angry, fearful, surprised, disgusted, and happy facial expressions) as the within-subject factor. As in Experiment 1, the statistical analysis was performed by a MANOVA with Pillai-Bartlett $V$ as test statistics (Olson, 1976), followed by ANOVAs with Geisser-Greenhouse correction of degrees of freedom, on each muscle separately. The comparisons among means were evaluated by Tukey HSD, with $\alpha=0.05$.

\section{Results and discussion}

The omnibus MANOVA revealed a significant main effect of Stimulus, $V=0.3148$, $F(20,1080)=4.61, p<0.0001)$, which indicates that the facial expressions of stimuli evoked different facial EMG reaction patterns. No significant main or interaction effects including the Sex of subject factor were revealed. Given these results, the Stimulus effect was examined by separate ANOVAs on each facial muscle.

The ANOVA for corrugator supercilii showed a significant effect of Stimulus. $F(4.0,217.6)=12.74, p<0.0001, \mathrm{MSe}=0.0131$. The means are illustrated in Fig. 5. As expected from the hypothesis that facial expressions are contagious, sad and angry faces evoked significantly larger responses compared to faces expressing happiness, disgust, and surprise, as indicated by the HSD test (HSD $=0.062$, df 6, 217). Stimuli of faces expressing fear evoked statistically greater reactions compared to happy stimuli.

The ANOVA for zygomatic major revealed a significant effect of Stimulus, $F(1.9,104.4)=7.62, p=0.0011, \mathrm{MSe}=0.0365$. The means are shown in Fig. 6. As predicted, happy faces evoked the largest zygomatic major response, which was significantly larger compared to all other stimuli, as indicated by the HSD test (HSD $=0.105$, df 6, 102). 


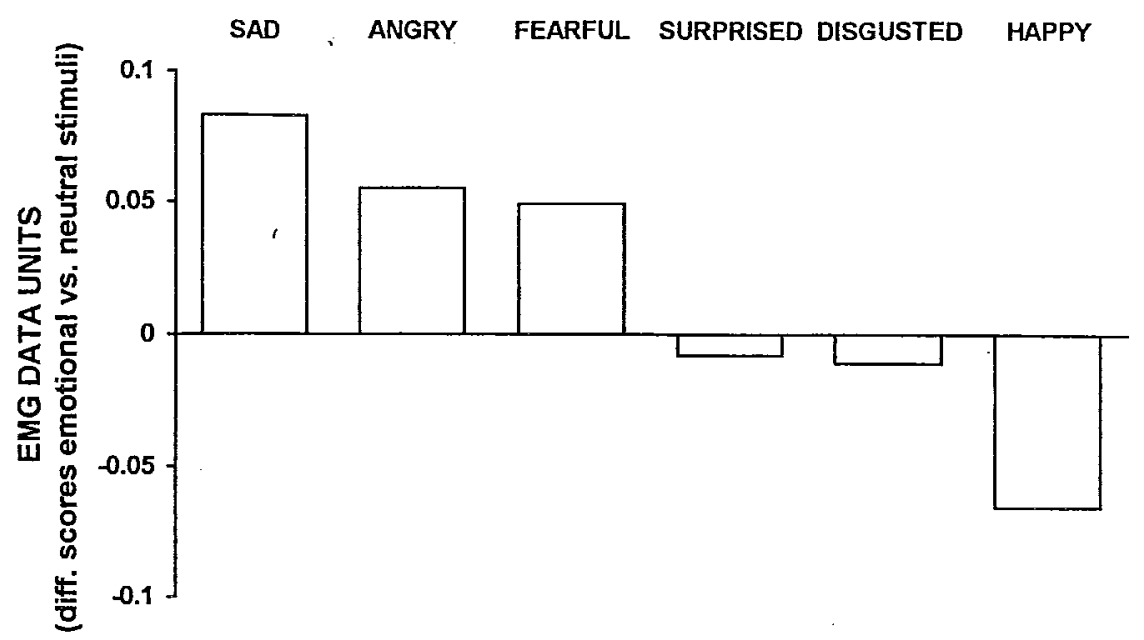

Fig. 5. The mean facial EMG response from the corrugator supercilii muscle region to stimuli of facial expressions obtained in Experiment 2.

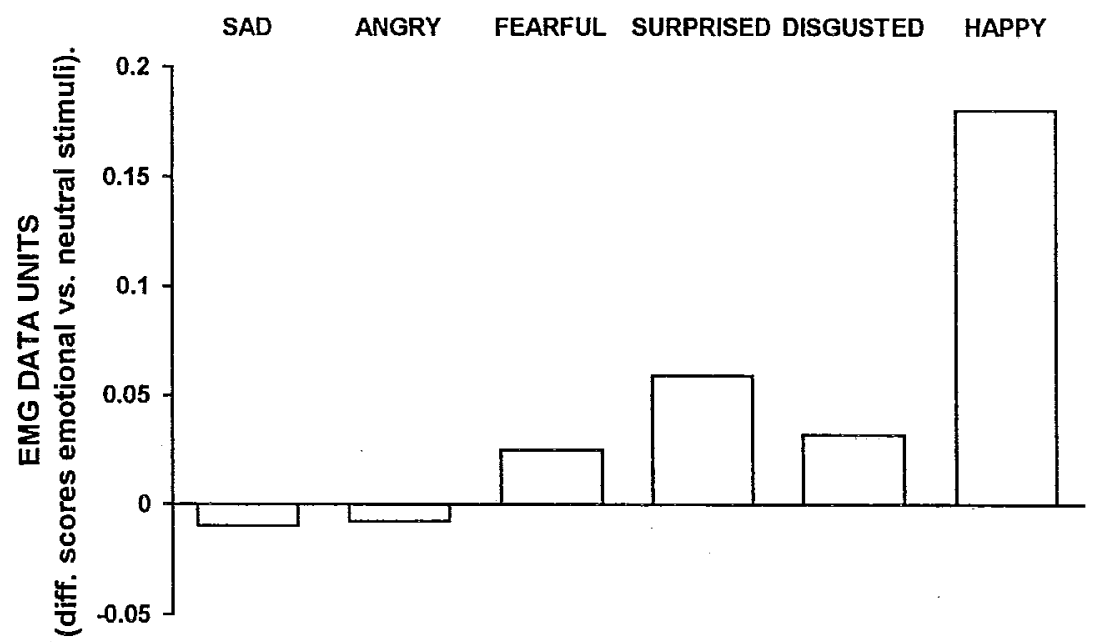

Fig. 6. The mean facial EMG response from the zygomatic major muscle region to stimuli of facial expressions obtained in Experiment 2.

Furthermore, the ANOVA for levator labii showed a significant effect of Stimulus, $F(3.4,188.4)=3.41, p=0.0144$, MSe $=0.0077$. As can be seen in Fig. 7 , stimuli of faces expressing disgust and happiness tended to evoke increased EMG activity. Unexpectedly, happy faces evoked significantly larger EMG responses compared to sad and angry faces, whereas faces expressing disgust did not evoke significantly larger EMG responses to any stimuli, as indicated by the HSD test (HSD $=0.048$, $\mathrm{df} 6,188$ ). This result may suggest that the levator labii muscle is used when forming a smile. However, this explanation is not supported by facial anatomy theory (Hjortsjö, 1970) unless, as Hjortsjö (1970) suggests, the levator labii muscle is activated in hearty laughter. There were no indications that subjects laughed at any stimuli and certainly not with hearty laughter. Therefore, the most plausible explanation is that the reactions recorded from the levator labii region are inflated by crosstalk from the zygomatic major muscle, as suggested by a positive correlation between these muscles $\left(r_{x y}=0.63, p<0.001\right)$. However, it is not likely that the levator labii activity may have caused the results obtained from the zygomatic major region, because zygomatic major differentiated happy from disgust faces, whereas the levator labii did not. 


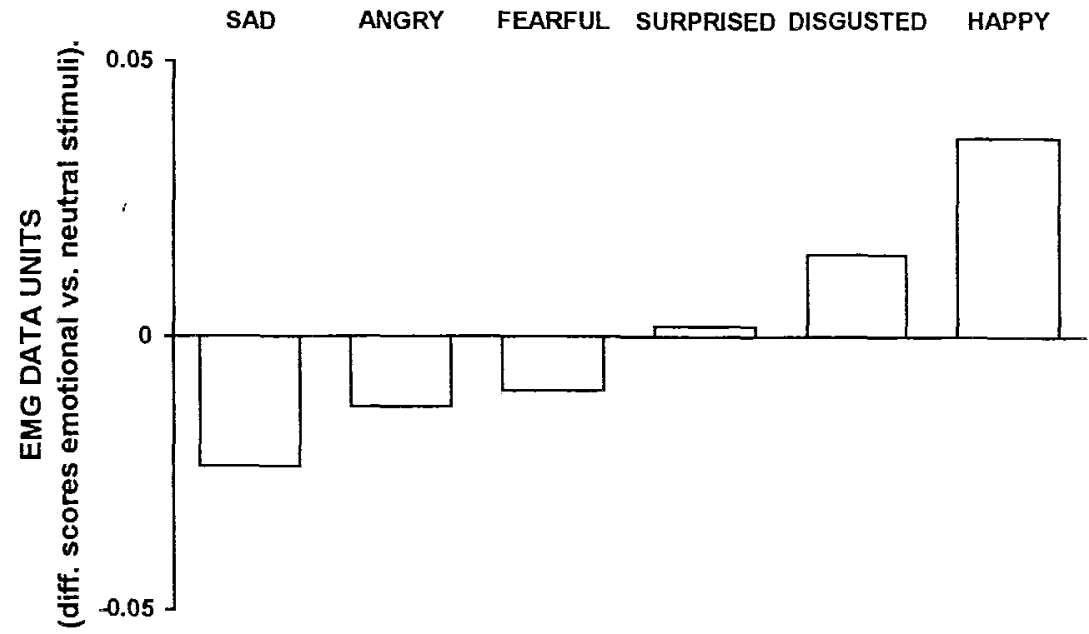

Fig. 7. The mean facial EMG response from the levator labii muscle region to stimuli of facial expressions obtained in Experiment 2.

Finally, the ANOVA for lateral frontalis showed a significant effect of Stimulus, $F(3.9,215.2)=2.43, p<0.05, \mathrm{MSe}=0.0111$ and the means are illustrated in Fig. 8. As expected from the facial emotional contagion hypothesis, faces expressing surprise evoked the largest mean EMG response, which was significantly larger compared to happy stimuli, as indicated by the HSD test (HSD $=0.058$, df 6,215 ).

No significant main or interaction effects including Sex of subjects were obtained by the MANOVA. An additional analysis, similar to that conducted in Experiment 1, did not reveal any significant gender differences in corrugator supercilii or zygomatic major response intensity to the stimuli of angry and happy faces.

To summarize the results of Experiment 2, it was revealed that, except for the levator labii responses to happy faces, all facial EMG responses obtained were consistent with the hypothesis that facial expressions are contagious.

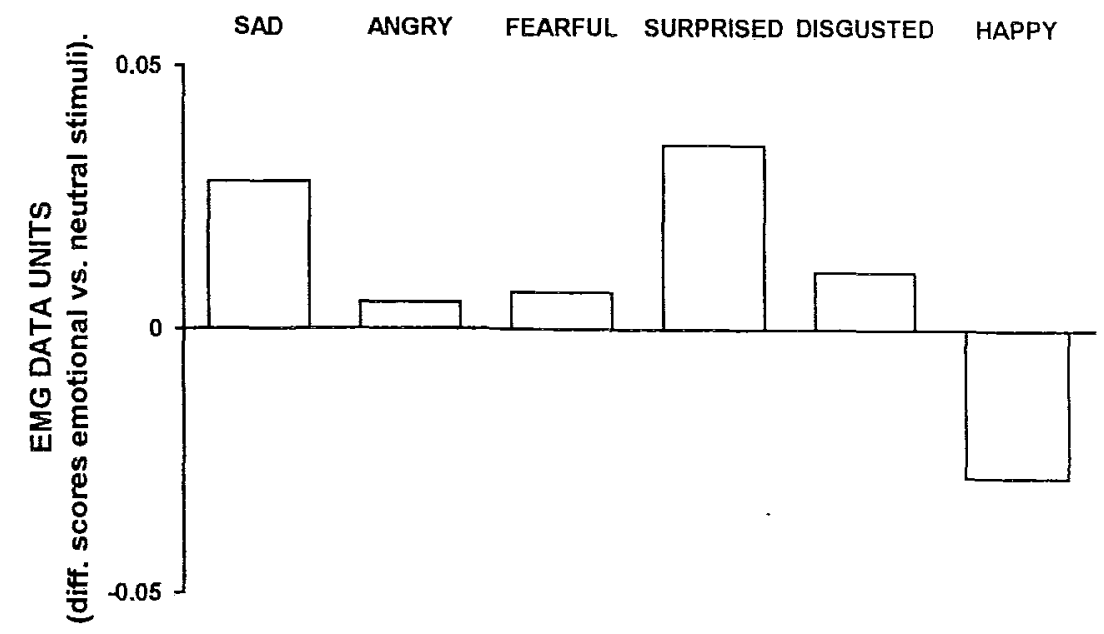

Fig. 8. The mean facial EMG response from the lateral frontalis muscle region to stimuli of facial expressions obtained in Experiment 2. 


\section{GENERAL DISCUSSION}

The results from both the experiments revealed that stimuli of facial expressions of emotion spontaneously elicited different facial EMG response patterns. Consistent with the hypothesis that facial expressions are contagious, stimuli of happy faces elicited EMG reactions characterized by increased zygomatic major activity, surprised faces with increased lateral frontalis activity, and finally sad, angry, and fearful faces were characterized by increased corrugator supercilii activity. The results obtained in Experiment 1 were in major parts replicated in Experiment 2, and the responses from the corrugator supercilii and zygomatic major muscle regions replicated those obtained in earlier studies using stimuli of angry and happy faces (Dimberg, 1982, 1988, 1990a, 1991; Dimberg \& Lundqvist, 1990), and stimuli of faces expressing anger, fear and happiness (McHugo et al., 1985).

Some results not inherent in the facial emotional contagion hypothesis were found and explained as artifacts of inter-site muscular crosstalk. Particularly the electrodes attached over the depressor supercilii and the levator labii muscle regions may be plagued by crosstalk from the corrugator supercilii and the zygomatic major muscle, respectively. However, can cross-talk also explain the results obtained from the lateral frontalis, the corrugator supercilii, and the zygomatic major muscle regions? Considering that corrugator supercilii differentiated angry from happy and surprised faces, zygomatic major happy from angry and surprised faces, and finally lateral frontalis differentiated surprised from happy and angry faces, it is reasonable to conclude that the EMG responses from these facial muscle regions are due to the emotional quality of the stimuli rather than to cross-talk artifacts among these muscles. Thus, from a methodological point of view, the present data suggests that the simple exposure paradigm, including the slide viewing technique together with facial EMG procedures, is a suitable paradigm for future exploration of facial emotional contagion. However, a larger number of facial muscle recording sites and better techniques for diminishing crosstalk artifacts would probably improve its applicability.

The aspects of gender differences in facial EMG intensity found in Experiment 1, which parallels the results reported by Dimberg and Lundqvist (1990), give some support, though modest, for the hypothesis that females are more susceptible to facial emotional contagion.

Although some of the results were inconsistent with the hypothesis that facial expressions are contagious, the major result of the present study was the demonstration that subjects mimic the facial expressions displayed by the stimulus persons. The present study was not designed to investigate the mechanisms underlying mimicry. However, one explanation to the obtained mimicking behavior may be that the facial expression stimuli evoked corresponding emotional experiences which then were expressed facially. This assumption is supported by the fact that the facial expression stimuli used in the present study evoked facial EMG response patterns consistent with those elicited by comparable but imagined emotional stimuli (Fridlund et al.,1984; Schwartz et al., 1979; Schwartz et al., 1980; Schwartz et al., 1976). That is, Schwartz and coworkers found that imagined negative emotional events (e.g., sadness, anger, or fear) were associated with increased corrugator supercilii activity, whereas imagined positive emotional events (happiness) were associated with increased zygomatic major activity.

A second explanation is that the obtained mimicking behavior primarily serve a communicative purpose, which is independent of the emotional state of the responder (Bavelas et al., 1986, 1987). However, considering that merely posed facial expressions give rise to corresponding subjective emotional experiences (e.g., Duclos et al., 1989; Levenson et al., 1990), it has been proposed that feedback from the facial muscles may be an important mechanism for the occurrence of emotional contagion (Hatfield et al., 1992). 
In conclusion, the results of the present study, irrespective of whether the facial EMG responses primarily have an experiential or a communicative basis, reveal that several different universal facial expressions evoke EMG activity over specific facial muscle regions that, to a certain extent, is consistent with the hypothesis that facial expressions are contagious. This may further suggest that facial emotional contagion is a prominent phenomenon in face-to-face situations and that facial expressions per se can serve as a channel for emotional contagion to occur.

I would like to thank Ulf Dimberg for helpful comments and encouragement during the development and completion of this study. This study was supported by grants to UIf Dimberg from the Swedish Council for Research in the Humanities and Social Sciences. Two anonymous reviewers are gratefully acknowledged for comments on an earlier version of this paper.

\section{REFERENCES}

Bavelas, J. B., Black, A., Lemery, C. R. \& Mullett, J. (1986). "I show how you feel": Motor mimicry as a communicative act. Journal.of Personality and Social Psychology, 50, 322-329.

Bavelas, J. B., Black, A., Lemery, C. R. \& Mullett, J. (1987). Motor mimicry as primitive empathy. In N. Eisenberger \& J. Strayer (Eds.), Empathy and its development (pp. 317-338). New York: Cambridge University Press.

Buck, R. (1984) The communication of emotion. New York: Guilford Press.

Darwin, C. (1872). The expression of emotion in man and animals. London: Murray.

Dimberg, U. (1982). Facial reactions to facial expressions. Psychophysiology, 19, 643-647.

Dimberg, U. (1988). Facial electromyography and the experience of emotion. Journal of Psychophysiology, 3, 277-282.

Dimberg, U. (1990a). Facial electromyography and emotional reactions. Psychophysiology, 27, 481-494.

Dimberg, U. (1990b). Facial reactions to auditory stimuli: Sex differences. Scandinavian Journal of Psychology, 31, 228-233.

Dimberg, U. (1991). Emotional reactions to facial expressions: A case of automatic responding? Psychophysiology, 28, S19, (abstract).

Dimberg, U. \& Christmanson, L. (1991). Facial reactions to facial expressions in subjects high and low in public speaking fear. Scandinavian Journal of Psychology, 32, 246-253.

Dimberg, U. \& Lundqvist, L.-O. (1990). Gender differences in facial reactions to facial expressions. Biological Psychology, 30, 151-159.

Duclos, S. E., Laird, J. D., Schneider, E., Sexter, M., Stern, L. \& Van Lighten, O. (1989). Emotion-specific effects of facial expressions and postures on emotional experience. Journal of Personality and Social Psychology, 57, 100-108.

Ekman, P. (1972). Universals and cultural differences in facial expressions of emotion. In J. K. Coles (Ed.) Nebraska Symposium on Motivation, 1971 (Vol. 19, pp. 207-283). Lincoln: University of Nebraska Press.

Ekman, P. (1989). The argument and evidence about universals in facial expressions of emotion. In $\mathrm{H}$ Wagner and A. Manstead (Eds.), Handbook of Social Psychophysiology (pp. 144-164). Chichester: John Wiley.

Ekman, P. \& Friesen, W. (1976). Pictures of facial affect. Palo Alto: Consulting Psychologists Press.

Fridlund, A. J. \& Cacioppo, J. T. (1986). Guidelines for human electromyographic research. Psychophysiology, 23, 567-589.

Fridlund, A. J., Ekman, P. \& Oster, H. (1987). Facial expressions of emotion: Review of literature, 1970-1983. In A. W. Siegman \& S. Feldstein (Eds.), Nonverbal behavior and communication ( $\mathrm{pp}$. 143-224). Hillsdale, NJ: Lawrence Erlbaum Associates.

Fridlund, A. J., Schwartz, G. E. \& Fowler, S. C. (1984). Pattern recognition to self-reported emotional state from multiple-site facial EMG activity during affective Imagery. Psychophysiology, 21, 622-637.

Hall, J. A. (1978). Gender effects in decoding nonverbal cues. Psychological Bulletin, 85, 845-857.

Hatfield, E., Cacioppo, J. T. \& Rapson, R. (1992). Primitive emotional contagion. In M. S. Clark (Ed.), Emotion and social behavior. Review of personality and social psychology (Vol. 14, pp. 151-177). Newbury Park, CA: Sage.

Hjortsjö, C.-H. (1970). Man's face and mimic language. Malmö: Studentlitteratur. 
Kirk, R. G. (1968). Experimental design: Procedures for the behavioral Sciences. Belmont, CA: Wadsworth.

Levenson, R. W., Ekman, P. \& Friesen, W. V. (1990). Voluntary facial action generates emotion-specific autonomic nervous system activity. Psychophysiology, 27, 363-384.

Marañon, G. (1950). The Psychology of Gesture. The Journal of Nervous and Mental Disease, 112, 469-497.

McDougall, W. (1928). An introduction to social psychology (21st ed.), London: Methuen.

McHugo, G. J., Lanzetta, J. T., Sullivan, D. G., Masters, R. D. \& Englis, B. G. (1985). Emotional reactions to a political leader's expressive displays. Journal of Personality and Social Psychology, 49, $1513-1529$.

Olson, C. L. (1976). On choosing a test statistic in multivariate analysis of variance. Psychological Bulletin, 83, 579-586.

Ribot, T. (1897). La psychologie des sentiments. Paris: Felix Alcan.

Schwartz, G. E., Ahern, G. L. \& Brown, S.-L. (1979). Lateralized facial muscle response to positive and negative emotional stimuli. Psychophysiology, 16, 561-571.

Schwartz, G. E., Brown, S.-L. \& Ahern, G. L. (1980). Facial muscle patterning and subjective experience during affective imaginary: Sex differences. Psychophysiology, 17, 75-82.

Schwartz, G. E., Fair, P. L., Salt, P., Mandel, M. R. \& Klerman, G. L. (1976). Facial muscle patterning to affective imagery in depressed and nondepressed subjects. Science, 192, 489-491.

Smith, Adam (1759/1976). The theory of moral sentiments. Edited by D. D. Raphael \& A. L. Macfie. Oxford: Clarendon Press.

Spencer, H. (1881). The principles of Psychology (3rd ed.). Vol. 2. London: Williams and Norgate.

Received 2 August 1993

Accepted 2 May 1994 